\title{
Correction par la fertilisation minérale des effets de l'ennoyage sur le blé d'hiver. I. - Expérimentation sur sol
}

\author{
Christian GUYOT \& Jean-Louis PRIOUL $\left({ }^{*}\right)$ \\ Rhône-Poulenc Recherches, Centre de Recherches de la Croix de Berny, 182, avenue A.-Briand, \\ F 92160 Antony
}

(*) Adresse actuelle : Laboratoire Structure et Métabolisme des Plantes, associé au C.N.R.S. (L.A. 40), Bâtiment 490, Université Paris-Sud, F 91405 Orsay Cedex

Les effets de l'ennoyage sur la croissance et la morphogenèse du blé d'hiver au tallage ont été étudiés en vue d'analyser l'efficacité d'une correction au moyen de la fertilisation minérale. Les plantes sont cultivées sur colonnes de terre en conditions contrôlées et l'ennoyage est appliqué au stade 2-3 feuilles pendant une semaine. Les effets sont observés 2 à 3 semaines après rétablissement du drainage.

Un apport d'engrais complet correspondant à $100 \mathrm{~kg}$. ha ${ }^{-1}$ de $\mathrm{N}, \mathrm{P}_{2} \mathrm{O}_{5}$ et $\mathrm{K}_{2} \mathrm{O}$ limite considérablement les conséquences de l'ennoyage. L'action est d'autant plus efficace que l'application est effectuée plus précocement par rapport à la contrainte. Le diagnostic foliaire montre le rôle essentiel de l'azote puis, en second, du phosphore, ce qui explique la supériorité des formulations NPK.

L'effet correcteur de l'engrais se manifeste surtout sur l'appareil aérien en limitant la réduction de croissance due à la carence minérale. Cette action quantitative ne modifie en rien la vitesse d'apparition des feuilles et des talles dont on confirme l'indépendance vis-à-vis de tous les facteurs externes hormis la température. Les conséquences pratiques de cette réponse sont discutées.

La croissance racinaire, fortement réduite par l'ennoyage, est au contraire stimulée aux dépens des parties aériennes après levée de la contrainte. Cet effet est favorisé par l'apport d'engrais.

Mots clés additionnels : Asphyxie racinaire, croissance, morphogenèse.

Alleviation of the consequences of waterlogging in winter wheat seedlings by mineral fertilization. I. - Soil culture experiments.

The effects of waterlogging on growth and morphogenesis were investigated in winter wheat at tillering in order to evaluate the correcting effect of fertilizer supply. Plants were grown on soil columns in a controlled environment and flooded for one week when the third leaf emerged. Results were observed two or three weeks after the relief of the constraint. A fertilizer supply equivalent to $100 \mathrm{~kg}$. ha ${ }^{-1} \mathrm{~N}, \mathrm{P}_{2} \mathrm{O}_{5}$ and $\mathrm{K}_{2} \mathrm{O}$ greatly aneviated waterlogging damage. The earlier the application before stress, the more efficient was the correcting effect. Mineral content analysis of the shoots during and after the stress showed the principal role of nitrogen, and to a lesser extent of phosphorus, in growth rate variation. These observations are consistent with the efficiency in the field of the NPK composition used. Fertilizer application had the greatest effect on the shoots, bv limiting the growth restriction induced by the decrease in mineral content. The rate of leaf and tiller development was independent of all external factors except temperature, and remained unaffected by the quantitative action of mineral nutrition on growth. The practical consequences of this response are discussed. By contrast, the growth of roots, severely restricted by waterlogging, was strongly stimulated after relief of the stress. This stimulation was greater than that of the shoots and was favoured by fertilization.

Additional key words : Root anaerobiosis, growth, morphogenesis. 


\section{INTRODUCTION}

Malgré une augmentation moyenne régulière $\left(100 \mathrm{~kg} . \mathrm{ha}^{-1} \cdot \mathrm{an}^{-1}\right)$, les rendements de blé tendre d'hiver présentent en France des variations interannuelles importantes et toujours très fortement liées aux fluctuations du climat (GUÉRIF, 1983). Les données climatiques de la période du $1^{\text {er }}$ septembre au 28 février expliquent à elles seules, pour de nombreux départements, plus de 60 p. 100 de la variabilité des rendements (DAGNEAUD et al., 1981). Les causes de ce comportement statistique sont complexes à analyser, mais il paraît clair que les conditions de sol, liées en particulier à des précipitations excessives, jouent un rôle important au moment où se réalise la structure du peuplement. L'ennoyage du sol, phénomène fréquent en mauvaise saison, représente en particulier une contrainte considérable pour la croissance. Les racines se trouvent en situation d'aération insuffisante à cause de la diffusion trop lente de l'oxygène dans la phase liquide, ce qui limite la respiration cellulaire (MORISSET et al., 1982). A court terme, cette contrainte conduit principalement à une restriction de croissance, puis à des symptômes de carence minérale dans les feuilles (DUTHION \& MINGEAU, 1976, TROUGHT \& DREW, 1982). Les effets sont d'autant plus négatifs que le niveau de fertilité du sol est plus bas. Ces observations ont suggéré un mode d'intervention corrective, basé sur un renforcement de la nutrition minérale, notamment azotée (SHALHEVET \& ZWERMAN, 1962 ; Stengel et al., 1972 ; TROught \& DREW, $1980 \mathrm{~b}$ ). Certains symptômes complémentaires comme la sénescence prématurée des feuilles âgées peuvent s'interpréter par des altérations de la biosynthèse racinaire de cytokinines, mais l'application foliaire de ces hormones a des effets correctifs assez limités (DREW et al., 1979, orge ; JACKSON \& CAMPBELL, 1979, tomate).

Une autre modification morphologique est cependant plus importante : celle du système racinaire, avec le surdéveloppement de racines adventives contenant des lacunes gazeuses importantes ou aérenchymes (JACKSON, 1955). Ce système, dans lequel une diffusion en phase gazeuse de l'oxygène est possible, est dépendant, au moins chez certaines espèces telles que le maïs, de la biosynthèse d'éthylène (DREW, 1983).

L'asphyxie racinaire par ennoyage est une contrainte climatique pour beaucoup de céréales d'hiver dont le blé. Dans le présent travail, l'objectif a été de reprendre, à un stade précis et important sur le plan pratique (Stade B, échelle de JONARD) l'étude des effets de l'ennoyage par l'analyse des possibilités et des limites d'une correction par renforcement de la fertilisation et en insistant sur un aspect peu abordé dans les travaux antérieurs : la réponse post-asphyxique. Cette $1^{\text {re }}$ étude, menée dans des conditions permettant le lien avec les observations au champ, a été prolongée par une analyse physiologique en conditions plus contrôlées (culture hydroponique, GUYOT \& PRIOUL, 1985) dans le but d'évaluer les processus limitant la croissance à l'échelle d'une plante entière ennoyée et d'envisager une intervention raisonnée sur des bases physiologiques correctes. Dans le présent travail, nous avons décrit l'effet de l'engrais sur la croissance, pendant et après l'ennoyage, puis la stratégie d'apport (date, composition) a été envisagée.

\section{MATÉRIEL ET MÉTHODES}

Les plantes sont cultivées dans des cylindres de PVC $(\varnothing: 6,7 \mathrm{~cm} ; \mathrm{h}: 30 \mathrm{~cm})$ contenant environ $1 \mathrm{~kg}$ de terre. Un écoulement est ménagé au bas de la colonne; le sol utilisé est un limon battant en provenance d'un champ expérimental (Avrainville, Essonne). En début d'essai, les colonnes remplies de terre séchée à l'air sont humidifiées par $300 \mathrm{~cm}^{3} \cdot \mathrm{kg}^{-1}$ d'eau (86 p. 100 de la saturation).

Les grains de blé, variété «Fidel» (provenance Ringot), sont imbibés dans de l'eau soumise à une aération constante, pendant une nuit à température ambiante ; 4 grains sont placés sous $1 \mathrm{~cm}$ de terre dans chaque colonne et disposés dans une enceinte climatique à $15-16^{\circ} \mathrm{C}$ sous un éclairement de 250 à $300 \mu \mathrm{E} . \mathrm{m}^{-2} \cdot \mathrm{s}^{-1}$, pendant $12 \mathrm{~h}$ par jour. Après levée, une seule plante par pot est conservée de manière à obtenir une population de hauteur homogène par élimination des plantes les plus grandes ou les plus petites. Les plantes sont arrosées chaque jour avec de l'eau, le volume ajouté correspondant aux pertes mesurées par pesée.

$\mathrm{Au}$ moment de l'ennoyage, réalisé 7 à $20 \mathrm{j}$ après la mise en germination, les colonnes de terre sont remplies d'eau par l'orifice inférieur de manière à chasser l'air. Le niveau d'eau est maintenu à $1 \mathrm{~cm}$ au-dessus du sol par un système de trop-plein.

Les masses de matière fraîche de chacune des feuilles et des talles sont pesées séparément. Les racines sont séparées de la terre par lavage à l'eau courante sur un tamis. Les longueurs et largeurs des $1^{\text {res }}$ feuilles sont mesurées. Le stade phénologique, repéré par le nombre de feuilles sur l'axe principal, est noté ainsi que le nombre et le rang des talles. Les masses de matière sèche des racines, feuilles et talles sont obtenues après passage à l'étuve à $65^{\circ} \mathrm{C}$ jusqu'à masse constante. La composition minérale (macro et oligoéléments) de ce matériel est déterminée en séparant racines et parties aériennes. Pour chaque variable mesurée, la signification statistique des traitements est obtenue par analyse de variance et comparaisons orthogonales selon le dispositif expérimental adopté.

\section{RÉSULTATS}

\section{A. Effet d'un apport d'engrais complet sur la crois- sance pendant et après ennoyage}

Un jour avant l'ennoyage, l'engrais est apporté en surface sous forme d'une solution à raison de $25 \mathrm{ml}$ par pot $\left(\mathrm{KNO}_{3}: 3,70 \mathrm{~g} .1^{-1} ; \mathrm{NH}_{4} \mathrm{NO}_{3}: 2,49 \mathrm{~g} . \mathrm{l}^{-1}\right.$; $\left.\mathrm{NH}_{4} \mathrm{H}_{2} \mathrm{PO}_{4}: 2,52 \mathrm{~g} . \mathrm{I}^{-1}\right)$, ce qui correspond à une dose d'environ $100 \mathrm{~kg} \cdot \mathrm{ha}^{-1}$ de $\mathrm{N}, \mathrm{P}_{2} \mathrm{O}_{5}$ et $\mathrm{K}_{2} \mathrm{O}$. L'ennoyage est maintenu une semaine à un stade précis pour chaque expérience (généralement feuille $n^{\circ} 3$ étalée). Des prélèvements hebdomadaires sont effectués jusqu'à 2 ou 3 semaines après le rétablissement du drainage. 


\section{Croissance}

A la fin de l'ennoyage (1 semaine), on observe une réduction de croissance des parties aériennes et des racines (encart fig. 1). Pour les feuilles, les écarts sont plus marqués en matière fraîche qu'en matière sèche en raison d'une plus faible teneur en eau des plantes ennoyées ( -10 à -15 p. 100). L'engrais a un effet légèrement bénéfique sur la croissance des parties aériennes, mais c'est surtout 2 à 3 semaines après le rétablissement du drainage que les différences s'expriment le plus nettement (fig. 1A et B). Les effets sont plus marqués lorsque la contrainte est appliquée au stade 3 feuilles $\left(21-25^{\mathrm{e}} \mathrm{j}\right)$ qu'au stade 1 feuille $\left(7^{\mathrm{e}} \mathrm{j}\right.$, fig. 1 et tabl. 1).

En raison de la variabilité des résultats, spécialement ceux de la croissance racinaire, les expériences d'ennoyage au stade 3 feuilles ont été répétées au minimum 4 fois par des cultures successives au cours de l'année. L'analyse des résultats (tabl. 1) montre que l'ennoyage réduit plus les masses de matière frâ̂che ou sèche des parties aériennes quand l'engrais est omis ( -40 contre -25 p. 100 en présence d'engrais). Au niveau des racines, la variabilité importante d'une culture à l'autre rend non significatifs la plupart des effets. Le pourcentage de racines est toutefois significativement supérieur après ennoyage. L'effet engrais se manifeste par une augmentation de la croissance des parties aériennes, partiellement au détriment des racines. Dans les racines des plantes ennoyées, les effets asphyxie et engrais se compensent (addition algébrique sans interaction significative) et aboutissent à une constante de la masse racinaire.

Malgré l'inégalité des contributions racinaires, on retrouve pour la croissance totale le même classement des traitements que pour les parties aériennes, avec des effets très significatifs de l'ennoyage et de l'engrais. Le pourcentage en masse de talles dans les parties
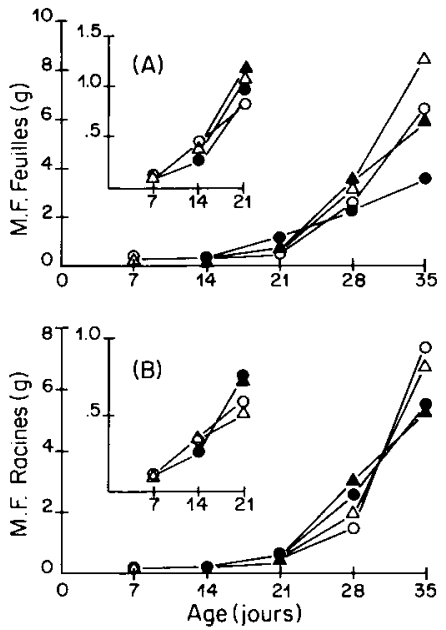

Figure 1

Evolution de la masse de matière fraîche des parties aériennes $(A)$ et des racines $(B)$ en fonction des traitements. Témoin avec $\triangle$ ou sans $\bigcirc$ engrais ; ennoyé du $7^{e}$ au $14^{e} j$ avec $\Delta$ ou sans $\bullet$ engrais ; chaque point représente la moyenne de 4 plantes et le trait vertical l'écart-type de la moyenne. Encart: agrandissement des $2^{e}$ et $3^{e}$ semaines.

Variation in shoot $(A)$ and root $(B)$ fresh weight after different treatments : control with $\triangle$ or without $\bigcirc$ fertilizer; waterlogged from day 7 to day 14 with $\mathbf{\Delta}$ or without - fertilizer; each point is the mean of four replicates, and vertical bar the standard error. Inset : enlargement of the second and third weeks.

aériennes est modifié par l'engrais ; cet effet est discuté plus complètement dans le paragraphe suivant.

\section{Morphogenèse foliaire}

En dépit de larges variations de taux de croissance selon les traitements (asphyxie et/ou engrais), on observe, à chaque prélèvement, une constance remarquable du stade foliaire atteint. Le suivi précis de

TABLEAU 1

Effet de l'ennoyage et de la fertilisation sur l'accumulation et la répartition de la matière fraîche et sèche chez le blé au stade jeune. Les plantes, soumises à une semaine d'ennoyage sont récoltées 2 semaines après le rétablissement du drainage (43-45 japrès germination). Moyennes de 12 plantes et analyse de variance des effets de l'asphyxie et de l'engrais (probabilité du test $F ; N S$ : différences non significatives au seuil de risque $5 \%$ ), pour 4 cultures successives.

Effect of waterlogging and fertilization on fresh and dry matter accumulation and partitioning in wheat at the young stage. Plants were flooded one week and harvested two weeks after relief of the stress (day 43-45 after germination). Means of 12 plants sampled from four independent experiments and two-way analysis of variance of waterlogging and fertilizer supply effects (probability of F-test; NS: non-significant at $5 \%$ probability level).

\begin{tabular}{|c|c|c|c|c|c|c|c|}
\hline & & \multicolumn{2}{|c|}{$\begin{array}{l}\text { Masse de matière fraîche } \\
(\mathrm{g})\end{array}$} & \multicolumn{2}{|c|}{$\begin{array}{l}\text { Masse de matière sèche } \\
(\mathrm{g})\end{array}$} & \multirow{2}{*}{$\begin{array}{c}\text { Racines } \\
\text { (\% masse de } \\
\text { matière fraîche } \\
\text { totale) }\end{array}$} & \multirow{2}{*}{$\begin{array}{c}\Sigma \text { Talles } \\
\text { (\% masse de } \\
\text { matière fraîche } \\
\text { des parties } \\
\text { aériennes) }\end{array}$} \\
\hline & & Parties aériennes & Racines & Parties aériennes & Racines & & \\
\hline \multirow{2}{*}{ Témoin } & sans engrais & 5,73 & 6,28 & 0,787 & 0,532 & 49,9 & 53,6 \\
\hline & avec engrais & 8,20 & 5,80 & 1,028 & 0,461 & 39,9 & 58,5 \\
\hline \multirow{2}{*}{ Ennoyé } & sans engrais & 3,46 & 4,97 & 0,463 & 0,415 & 56,2 & 49,8 \\
\hline & avec engrais & 6,14 & 5,79 & 0,765 & 0,417 & 47,4 & 57,3 \\
\hline Asphyxie & & $<0,01 \%$ & NS & $<0,01 \%$ & NS. & $0,4 \%$ & NS \\
\hline $\begin{array}{l}\text { Engrais } \\
\text { Asphyxie }\end{array}$ & & $<0,01 \%$ & NS & $<0,01 \%$ & NS & $0,02 \%$ & $<0,01 \%$ \\
\hline$\times$ Engrais & & NS & NS & NS & NS & NS & NS \\
\hline
\end{tabular}


l'élongation des feuilles successives du brin-maître montre un retard dans l'apparition des feuilles qui atteint un maximum de 2 à $3 \mathrm{j}$ dans le cas de la feuille 6 chez les plantes asphyxiées sans engrais. Ce retard initial a le plus souvent disparu au moment où les feuilles atteignent leur longueur maximale.

Le tallage se déroule comme dans la séquence décrite au champ par MASLE-MEYNARD \& SEBILLOTTE (1981): la talle de rang 1 devient visible audessus de la ligule de la $1^{\text {re }}$ feuille lorsque le brinmaître possède 4 feuilles et les talles successives sont émises au même rythme que les feuilles. Par rapport aux données précitées, les talles d'ordre supérieur $\left(T_{12}, T_{21}, T_{3 p}\right)$ apparaissent un peu plus tôt, comme l'indique MASLE-MEYNARD (1981). La somme de température pour chaque phyllochrone est un peu inférieure, 75-80 au lieu des 90-100 degrés $\times$ jours observés par MASLE-MEYNARD \& SEBILLOTE (1981).

Dans ce cadre morphogénétique constant, les différences pondérales importantes décrites plus haut se manifestent dans la taille des organes développés. Les feuilles en croissance au moment de l'ennoyage $\left(F_{2}, F_{3}\right)$ tendent à être plus petites en longueur et en largeur ( -10 p. 100). Ce fait est en rapport avec la baisse de teneur en eau qui, diminuant la turgescence des feuilles, freine leur élongation. L'effet principal des traitements apparaît au niveau des talles; la masse des talles primaires se réduit, sans que leur séquence soit affectée, quand la matière végétale disponible pour la croissance devient notablement limitante (ennoyés et/ou sans engrais); le nombre et la taille des talles secondaires diminuent et les talles d'ordre supérieur sont absentes. En aucun cas, la vitesse d'apparition des talles n'est modifiée. Cette réduction du tallage est-elle due à un effet spécifique des traitements sur la séquence d'apparition ou la réduction du nombre de talles provient-elle simplement de l'insuffisance de matière sèche disponible pour la croissance? La représentation de la masse de matière fraîche cumulée des talles en fonction de la masse de matière fraîche des parties aériennes montre (fig. 2) que, tous traite-

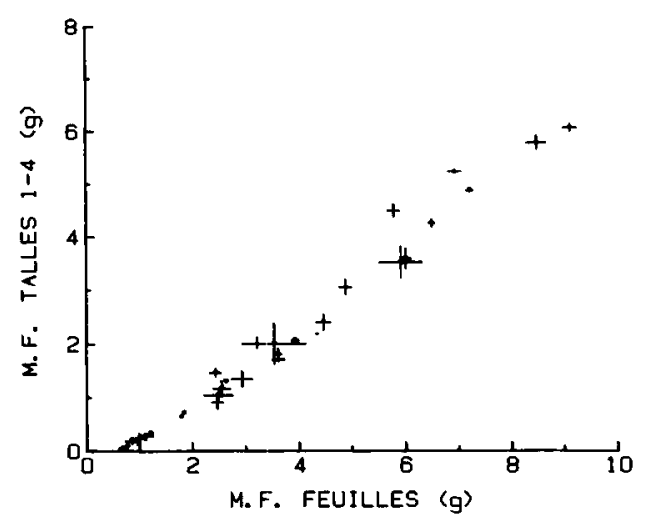

Figure 2

Masse de matière fraîche des talles 1 à 4 en fonction de la masse de matière fraîche des parties aériennes au cours de prélèvements hebdomadaires successifs à partir de l'application des traitements; chaque point représente la moyenne de 4 mesures correspondant à un prélèvement d'un traitement.

Tiller (1 to 4) fresh weight in relation to shoot fresh weight for plants harvested weekly after application of treatments; each point is the mean of four replicates. ments confondus, les points expérimentaux s'ajustent sur une seule droite à partir d'un seuil $0,7 \mathrm{~g}$. Les écarts observés dans les masses des talles en p. 100 de la masse aérienne (tabl. 1) sont compatibles avec la relation de la figure 2 et proviennent simplement du fait que la droite ne passe pas par l'origine des axes; il n'y a donc pas une proportionnalité exacte entre les 2 variables. La figure 2 démontre clairement que le mode de répartition des assimilats entre les différents organes aériens est indépendant des traitements appliqués. Comme, d'autre part, la variation du nombre de talles se fait seulement au niveau des talles d'ordre supérieur à 2, c'est-à-dire des moins prioritaires, il est
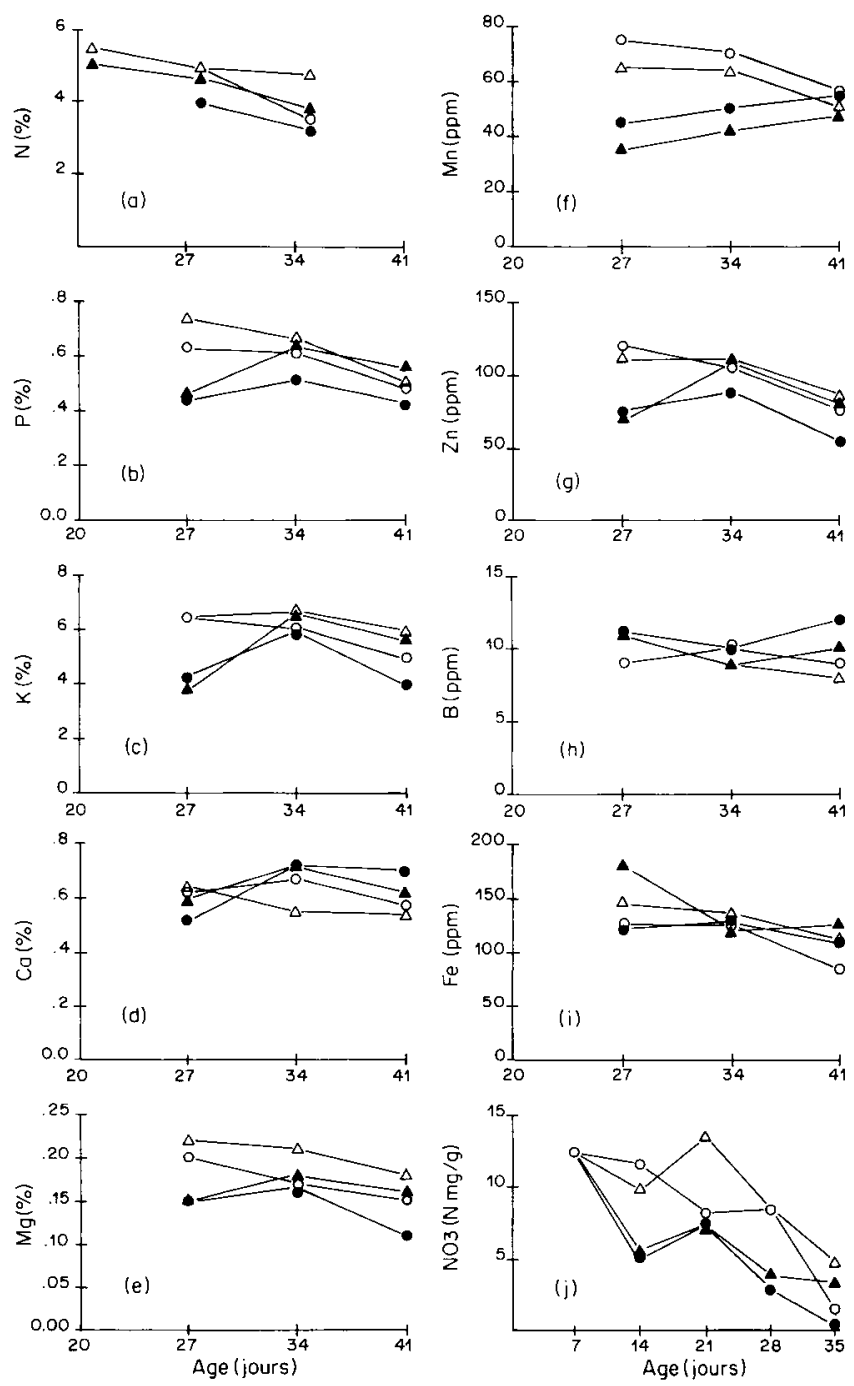

Figure 3

Teneur en éléments totaux: Azote (a), Phosphore (b), Potassium (c), Calcium (d), Magnésium (e), Manganèse (f), Zinc (g), Bore (h), Fer (i) et en Azote nitrique (j) des parties aériennes, rapportée à la masse de matière sèche (p. IOO ou partie par million (ppm). Témoin avec $\triangle$ ou sans $\bigcirc$ engrais; ennoyé avec $\Delta$ ou sans $\bullet$ engrais du $20^{e}$ au $27^{e} j$ sauf pour $\mathrm{NO}_{3}$ où les données proviennent d'une autre expérience avec ennoyage du $7^{e}$ au $14 \mathrm{j}$; moyennes de 4 mesures.

Mineral content of shoots, expressed on a dry matter basis (\% or parts per million (ppm) : Nitrogen (a), Phosphorus (b), Potassium (c), Calcium (d), Magnesium (e), Manganese (f), Zinc (g), Boron (h), Iron (i), Nitric nitrogen (j). Control with $\triangle$ or without $\bigcirc$ fertilizer; waterlogged with $\boldsymbol{\Delta}$ or without $\bullet$ fertilizer from day 20 to 27 except for $\mathrm{NO}_{3}$ where data were taken from another experiment with waterlogging from $7^{\text {th }}$ to $14^{\text {th }}$ day. Means of four replicates. 
logique de penser que, dans le cas présent, le tallage est contrôlé principalement par la quantité d'assimilats disponibles.

\section{Composition minérale}

Dans les témoins, les teneurs des parties aériennes en éléments majeurs ( $\mathrm{N}, \mathrm{P}, \mathrm{K}, \mathrm{Ca}, \mathrm{Mg}$ ) et en certains oligo-éléments $(\mathrm{Mn}, \mathrm{Zn}, \mathrm{Fe}$ ) baissent régulièrement selon le classique effet de dilution de la phase juvénile. Les traitements avec ennoyage et/ou absence d'engrais provoquent généralement une réduction beaucoup plus drastique. A la fin de l'ennoyage $\left(27^{\mathrm{e}} \mathrm{j}\right)$, les teneurs en éléments majeurs $(\mathrm{P}, \mathrm{K}, \mathrm{Mg})$ et en oligo-éléments $(\mathrm{Mn}, \mathrm{Zn})$ sont fortement réduites dans les parties aériennes des plantes ennoyées (fig. 3a à i). D'autres expériences non présentées montrent qu'il en est de même pour l'azote total ; le calcium, le bore et le fer font exception. L'apport d'engrais avant ennoyage a peu d'effet sur les teneurs à la sortie de la contrainte. L'arrêt de l'ennoyage permet au contraire, dans la semaine qui suit, une forte remontée des teneurs qui est renforcée par la présence d'engrais pour $\mathrm{N}, \mathrm{P}, \mathrm{K}$ et $\mathrm{Zn}$. Dans la $2^{\mathrm{e}}$ semaine, la tendance à la dilution reprend sans qu'il y ait toujours retour aux valeurs du témoin. Les plantes ennoyées accumulent plus de $\mathrm{Ca}, \mathrm{B}$ et $\mathrm{Fe}$ que les témoins. Comme pour les croissances, les effets d'une semaine d'ennoyage persistent plus de 2 semaines après la cessation de la cause ; ceci est particulièrement vrai pour l'azote (fig. 3a) et pour $\mathrm{K}, \mathrm{Mg}$ et $\mathrm{Zn}$ dans les plantes ennoyées sans engrais. L'évolution des concentrations en nitrate permet de décrire de façon beaucoup plus sensible que le p. 100 d'azote le comportement du métabolisme azoté (fig. $3 \mathrm{j}$ ) : l'apport d'engrais au $7^{\mathrm{e}} \mathrm{j}$ provoque, la semaine suivante, une augmentation de teneur dans les racines témoins ou traitées, effet retardé d'une semaine par la présence d'ennoyage. Dans les feuilles (fig. 3j), l'effet de l'engrais n'apparaît qu'après 2 semaines chez les témoins et 4 semai- nes chez les ennoyés. Les différences entre les 4 traitements s'amplifient après 3 et 4 semaines, ce qui correspond probablement à l'épuisement des réserves endogènes en nitrates dans les sols non complémentés en engrais.

\section{B. Effet de la date d'apport de l'engrais par rapport à celle de l'ennoyage}

L'engrais complet (cf. $\S$ A) est apporté selon 3 modalités : au moment du semis, au début de l'ennoyage ou immédiatement après. La récolte a lieu 2 semaines après rétablissement du drainage. Par comparaison avec les témoins non ennoyés où la date d'apport n'a pas d'influence significative sur la croissance aérienne ou racinaire, on observe chez les ennoyés une croissance d'autant plus réduite que l'addition d'engrais est plus tardive. Cette tendance est plus marquée au niveau racinaire (pourcentage significativement inférieur dans la masse de matière totale) (tabl. 2). Les compositions minérales des plantes à la récolte sont significativement différentes. Les valeurs après addition d'engrais au semis sont concordantes avec celles indiquées aux figures $3 a$ et $b$. L'apport après ennoyage tend à augmenter les teneurs en azote total et phosphore, particulièrement dans les lots ennoyés. Ceci est cohérent avec un temps d'utilisation plus court de l'engrais.

\section{Effet de la composition de l'engrais ( $N$ et/ou $P$ )}

Juste avant l'ennoyage, les plantes sont alimentées par $25 \mathrm{ml}$ de l'une des 4 solutions suivantes contenant une quantité identique de potassium et complétée ou non par de l'azote et/ou du phosphore $\mathrm{O}\left(2,55 \mathrm{~g} . \mathrm{l}^{-1} \mathrm{~K}_{2} \mathrm{SO}_{4}\right)$, N (2,96 g. $\mathrm{l}^{-1} \mathrm{KNO}_{3}, 3,77 \mathrm{~g} . \mathrm{I}^{-1}$ $\left.\mathrm{NH}_{4} \mathrm{NO}_{3}\right), \mathrm{P}\left(1,31 \mathrm{~g} . \mathrm{I}^{-1} \mathrm{KH}_{2} \mathrm{PO}_{4}, 1,72 \mathrm{~g} . \mathrm{I}^{-1} \mathrm{~K}_{2} \mathrm{HPO}_{4}\right)$, NP $\left(1,31 \quad\right.$ g.1 $1^{-1} \quad \mathrm{KH}_{2} \mathrm{PO}_{4}, \quad 1,72 \quad \mathrm{~g} .1^{-1} \quad \mathrm{~K}_{2} \mathrm{HPO}_{4}$ et $3,9 \mathrm{~g} .1^{-1} \quad \mathrm{NH}_{4} \mathrm{NO}_{3}$ ). Une semaine d'ennoyage au stade 2 feuilles est suivie de 2 semaines avec drainage normal à l'issue desquelles les plantes sont récoltées.

TABLEAU 2

Effet de la date d'apport d'engrais complet sur l'accumulation de la matière sèche et la composition minérale de plantes témoins ou soumises à un ennoyage d'une semaine. L'engrais est incorporé au sol au semis ou épandu en surface, sous forme de solution, au début ou à la fin de l'ennoyage. La récolte est effectuée 2 semaines après le rétablissement du drainage (34 j après germination). Moyennes de 4 plantes et signification statistique de l'effet date d'apport de l'engrais (probabilité du test $F ; N S:$ différences non significatives au seuil de risque $5 \%$ ).

Effect of fertilizer application time on dry matter accumulation, partitioning and mineral content in control or flooded (one week) seedlings. The fertilizer was applied at sowing, hefore or after flooding. The plants were sampled two weeks after stress relief (day 34 after germination). Means of four plants and statistical comparison between dates of fertilizer application (probability of F-test; NS : non-significant at $5 \%$ probability level).

\begin{tabular}{|c|c|c|c|c|c|c|}
\hline & \multirow[t]{2}{*}{$\begin{array}{l}\text { Date d'apport } \\
\text { d'engrais }\end{array}$} & \multicolumn{2}{|c|}{$\begin{array}{l}\text { Masse de matière sèche } \\
(\mathrm{g})\end{array}$} & \multirow{2}{*}{$\begin{array}{c}\text { Racines } \\
(\% \text { masse } \\
\text { de matière sèche } \\
\text { totale })\end{array}$} & \multicolumn{2}{|c|}{$\begin{array}{c}\text { Composition minérale } \\
\text { des parties aériennes } \\
(\% \text { masse de matière sèche })\end{array}$} \\
\hline & & Parties aériennes & Racines & & Azote total & Phosphore \\
\hline \multirow{3}{*}{ Témoin } & Semis & 0,674 & 0,273 & 26,9 & 5,09 & 0,369 \\
\hline & Avant ennoyage & 0,719 & 0,302 & 27,9 & 4,80 & 0,370 \\
\hline & Après ennoyage & 0,701 & 0,274 & 25,0 & 5,04 & 0,395 \\
\hline \multirow{3}{*}{ Ennoyé } & Semis & 0,631 & 0,296 & 31,6 & 4,64 & 0,364 \\
\hline & Avant ennoyage & 0,568 & 0,211 & 27,6 & 4,72 & 0,381 \\
\hline & Après ennoyage & 0,532 & 0,172 & 23,6 & 5,25 & 0,501 \\
\hline Date d'apport & & NS & NS & $0,4 \%$ & $4,8 \%$ & $0,3 \quad \%$ \\
\hline
\end{tabular}


TABLEAU 3

Effet de la composition de l'engrais (N, P, NP) appliqué avant ennoyage d'une semaine, sur l'accumulation et la répartition de la matière fraîche et sèche, observé 2 semaines après rétablissement du drainage (34 j après germination). Moyennes de 6 plantes et analyse de variance des effets azote $(N)$ et phosphore $(P)$ de la composition de l'engrais (probabilité du test $F ; N S:$ différences non significatives au seuil de risque $5 \%$ ).

Effect of fertilizer composition (N, P, NP), applied before a one-week waterlogging, on fresh and dry matter accumulation and partitioning, observed two weeks after relief of the stress (dav 34 after germination). Means of six replicates and two-wav anatvsis of variance of nitrogen (N) and phosphorus $(P)$ effects in fertilizer composition (probability of F-test ; NS : non-significant at $5 \%$ probability level).

\begin{tabular}{|c|c|c|c|c|c|c|c|}
\hline & \multirow{2}{*}{ Engrais } & \multicolumn{2}{|c|}{$\begin{array}{l}\text { Masse de matière fraîche } \\
\qquad(\mathrm{g})\end{array}$} & \multicolumn{2}{|c|}{$\begin{array}{l}\text { Masse de matière sèche } \\
\text { (g) }\end{array}$} & \multirow{2}{*}{$\begin{array}{c}\text { Racines } \\
\text { (\% masse de } \\
\text { matière fraîche } \\
\text { totale) }\end{array}$} & \multirow{2}{*}{$\begin{array}{c}\text { Talles } \\
\text { (\%0 masse de } \\
\text { matière fraîche } \\
\text { des parties } \\
\text { aériennes) }\end{array}$} \\
\hline & & Parties aériennes & Racines & Parties aériennes & Racines & & \\
\hline \multirow{4}{*}{ Témoin } & $\mathrm{O}$ & 5,69 & 7,56 & 0,799 & 0,570 & 55,0 & 44,9 \\
\hline & $\mathrm{N}$ & 6,57 & 5,06 & 0,810 & 0,354 & 41,3 & 46,5 \\
\hline & $\mathbf{P}$ & 5,43 & 6,90 & 0,735 & 0,503 & 54,5 & 45,4 \\
\hline & NP & 8,22 & 7,24 & 1,087 & 0,510 & 45,7 & 46,7 \\
\hline \multirow{8}{*}{ Ennoyé } & $\mathrm{O}$ & 3,16 & 4,64 & 0,442 & 0,362 & 57,5 & 43,9 \\
\hline & $\mathrm{N}$ & 5,08 & 4,57 & 0,625 & 0,284 & 46,2 & 47,8 \\
\hline & $\mathbf{P}$ & 3,33 & 5,74 & 0,459 & 0,421 & 62,8 & 43,9 \\
\hline & NP & 5,96 & 6,73 & 0,775 & 0,447 & 52,2 & 46,2 \\
\hline & Asphyxie & $<0,01 \%$ & NS & $<0,01 \%$ & $4.20_{0}$ & $0.90_{0}^{\prime}$ & $<0,01 \%$ \\
\hline & $N$ & $<0,01 \%$ & NS & $<0,01 \%$ & NS & $<0,01 \%$ & $0,1 \%$ \\
\hline & $\mathrm{P}$ & $3,4 \%$ & NS & $4,2 \%$ & NS & NS & NS \\
\hline & $\mathbf{N} \times \mathbf{P}$ & $2,3 \%$ & NS & $1,2 \%$ & NS & NS & NS \\
\hline
\end{tabular}

\section{Croissance}

Apporté seul, l'azote augmente nettement la croissance aérienne ; en valeur relative, l'effet est sensiblement égal qu'il y ait ennoyage ou non (tabl. 3). L'action du phosphore n'est nettement positive qu'en présence d'azote, en particulier chez les témoins. L'interaction $\mathrm{N} \times \mathrm{P}$ est très significative. Au niveau des racines, aucun des effets $\mathrm{N}$ ou $\mathrm{P}$ n'est globalement significatif (tabl, 3). Quand on rapporte la variation des masses de racines à celle des masses de matière fraîche ou sèche totales, on confirme la baisse de la proportion de racines en présence d'azote et, au contraire, l'augmentation de cette proportion sous l'influence d'une asphyxie racinaire préalable sans que le phosphore intervienne. En croissance totale, les effets bénéfiques des apports de $\mathrm{N}$ et $\mathrm{P}$ sur les ennoyés sont du même ordre ; c'est leur combinaison

TABLEAU 4

Effet de la composition de l'engrais $(N, P, N P)$ appliqué avant ennoyage d'une semaine sur la teneur en éléments minéraux des systèmes foliaires et racinaires, mesurée 2 semaines après rétablissement du drainage (34 $j$ après germination). Movenne de 3 mesures et analyse de variance des effets azote $(N)$ et phosphore $(P)$ de la composition de l'engrais (probabilité du test $F ; N S:$ différences non significatives au seuil de risque $5 \%$.

Effect of fertilizer composition $(N, P, N P)$ applied before a one-week waterlogging, on mineral content in shoots and roots, two weeks after relief of the stress (day 34 after germination). Means of three replicates and two-way analysis of variance of nitrogen $(N)$ and phosphorus $(P)$ effects in fertilizer composition (probability of F-test; NS: non-significant at $5 \%$ probability level).

\begin{tabular}{|c|c|c|c|c|c|c|c|c|c|c|c|}
\hline \multicolumn{12}{|c|}{ Composition minérale ( $\%$ masse de matière sèche) } \\
\hline & & \multicolumn{2}{|c|}{$N$} & \multicolumn{2}{|c|}{$\mathbf{P}$} & \multicolumn{2}{|c|}{$\mathrm{K}$} & \multicolumn{2}{|c|}{$\mathrm{Ca}$} & \multicolumn{2}{|c|}{$\mathrm{Mg}$} \\
\hline & & Feuilles & Racines & Feuilles & Racines & Feuilles & Racines & Feuilles & Racines & Feuilles & Racines \\
\hline \multirow{4}{*}{ Témoin } & $\mathrm{O}$ & 2,94 & 1,64 & 0,370 & 0,313 & 4,76 & 3,24 & 0,44 & 0,94 & 0,11 & 0,21 \\
\hline & $N$ & 4,63 & 2,63 & 0,419 & 0,346 & 5,84 & 3,43 & 0,52 & 1,30 & 0,16 & 0,35 \\
\hline & $\mathbf{P}$ & 2,84 & 1,63 & 0,432 & 0,374 & 5,13 & 3,41 & 0,46 & 0,72 & 0,10 & 0,20 \\
\hline & NP & 3,96 & 2,17 & 0,459 & 0,349 & 5,28 & 3,12 & 0,47 & 0,94 & 0,14 & 0,29 \\
\hline \multirow{8}{*}{ Ennoyé } & $\mathrm{O}$ & 2,95 & 1,57 & 0,444 & 0,361 & 4,90 & 3,87 & 0,53 & 0,91 & 0,11 & 0,22 \\
\hline & $\mathrm{N}$ & 4,30 & 2,47 & 0,482 & 0,379 & 5,66 & 3,80 & 0,57 & 0,81 & 0,15 & 0,31 \\
\hline & $\mathrm{P}$ & 2,68 & 1,44 & 0,484 & 0,378 & 4,80 & 3,66 & 0,56 & 1,26 & 0,10 & 0,21 \\
\hline & NP & 3,64 & 2,09 & 0,499 & 0,386 & 5,18 & 3,47 & 0,53 & 0,97 & 0,13 & 0,26 \\
\hline & Asphyxie & NS & NS & $0,06 \%$ & $1,2 \%$ & NS & $0,1 \%$ & $<0,01 \%$ & NS & NS & NS \\
\hline & $N$ & $<0,01 \%$ & $<0,01 \%$ & $2,3 \%$ & NS & $0,08 \%$ & NS & NS & NS & $<0,01 \quad \%$ & $<0,01 \%$ \\
\hline & $\mathrm{P}$ & $2,1 \%$ & $3,5 \%$ & $0,7 \%$ & NS & NS & NS & NS & $1,6 \%$ & $2,7 \%$ & NS \\
\hline & $\mathbf{N} \times \mathbf{P}$ & NS & NS & NS & MS & $3,9 \%$ & NS & NS & NS & NS & NS \\
\hline
\end{tabular}


NP qui donne le résultat le plus favorable, proche des témoins avec apport incomplet d'engrais.

Comme dans les expériences précédentes, les masses des talles successives restent approximativement proportionnelles à celles des brins-maîtres (tabl. 3). Les effets sur les talles primaires ( 1 à 4 ) sont comparables à ceux observés sur la masse totale aérienne : effet positif de $\mathrm{N}$ accentué par la présence de $\mathrm{P}$, effet nul ou dépressif de $\mathrm{P}$ en absence d'azote.

\section{Composition minérale}

Dans l'ensemble, après 2 semaines de récupération, les effets de l'ennoyage sont très peu marqués. Les exceptions les plus notables sont celles du phosphore (parties aériennes et racines), du potassium racinaire, $\mathrm{du}$ calcium foliaire, pour lesquels les concentrations sont supérieures dans les plantes ayant subi l'ennoyage. Indépendamment de l'asphyxie, l'apport séparé d'azote ou de phosphore augmente logiquement les teneurs de ces 2 éléments, mais leur addition simultanée tend à diminuer la teneur en azote (tabl. 4), probablement en raison d'un effet de dilution dû à la plus forte stimulation de la croissance.

\section{DISCUSSION}

La restriction très brutale de la croissance racinaire, le ralentissement de la croissance foliaire et l'apparition d'une carence minérale importante sous l'effet de l'ennoyage que nous avons décrits confirment les résultats antérieurs obtenus sur le blé (TROUGHT \& DREW, $1980 a$ et $b$ ) ou d'autres espèces (DUTHION \& MingeAU, 1976 ; DREW et al., 1979). L'étude de la phase de récupération après rétablissement du drainage sur laquelle nous avons porté notre effort, permet de préciser que les plantes ennoyées ont une croissance modifiée même 2 semaines après suppression de la contrainte. Le retard de croissance est plus marqué au niveau aérien que souterrain, alors que c'est l'inverse immédiatement après la phase d'ennoyage ; la reprise se montre donc d'abord au niveau racinaire.

Un apport d'engrais limite considérablement les conséquences de l'ennoyage. Il est d'autant plus efficace que l'application se fait sous une formulation plus complète $(\mathrm{N}, \mathrm{P}, \mathrm{K})$. En particulier, l'apport de phosphate soluble en même temps que les nitrates empêche l'apparente carence en phosphore qu'observaient TROUGHT \& DREW (1980b) quand les nitrates sont appliqués seuls. L'intérêt d'une application avant et pendant la contrainte est à souligner.

La compréhension des effets à long terme d'une contrainte telle que l'ennoyage nécessite, en plus des critères de croissance pondérale objets de la plupart des investigations, l'analyse des conséquences sur le développement. De ce point de vue, nos résultats dẹmontrent pour la première fois très clairement que l'ennoyage n'a pratiquement aucune action sur la vitesse d'apparition des talles et des feuilles du blé au stade $B$. Ceci apporte un degré de généralité plus grand aux observations faites au même stade par MASLE-MEYNARD (1981 et 1982) sur des plantes au champ en compétition pour l'azote. La complète indépendance de la vitesse de développement à l'égard des facteurs externes hormis la température est remarquable. Un autre invariant mis en évidence est la répartition de la matière végétale entre le brin-maitre et les talles: la masse de matière affectée aux talles ne dépend que de la masse totale à répartir et non du nombre de talles possibles. Les contraintes externes introduites par les traitements n'agissent que par l'intermédiaire de leur action sur la croissance totale.

Les conséquences pratiques de ce comportement sont importantes. En effet, la différenciation des épis suit un rythme très voisin sur le brin-maître et les 2 ou 3 premières talles, mais cette différenciation n'a lieu que si ces talles ont atteint une masse de matière sèche et un nombre de feuilles minimaux (MASLE-MEYNARD, 1981). Si, au moment de cette période critique, la croissance des talles est inférieure à ce minimum, les épis se forment uniquement sur le brin-maître.

Un élément supplémentaire intervient dans les cas de l'ennoyage. En effet, celui-ci restreint plus fortement la croissance des racines que celle des parties aériennes; c'est l'inverse qui se produit lors de la reprise après ennoyage : on assiste à un surdéveloppement racinaire au détriment du système aérien; la plus faible quantité de matière sèche disponible diminue alors le nombre de talles aptes à se développer bien que les traitements n'aient aucun effet sur la vitesse d'apparition des feuilles et des talles.

Ces constatations conduisent à formuler la nécessité d'intervention pendant le stress de façon à préserver le minimum (un brin-maître et 2 ou 3 talles) qui permettra la réalisation des objectifs de rendement. Un renforcement de la nutrition minérale apparaît le moyen efficace le plus immédiat; il faut logiquement en rechercher la cause par une expérimentation plus précise en conditions hydroponiques et en explorer les possibilités pratiques par des essais au champ. De ce point de vue, les enquêtes entreprises dans le cadre de l'implantation du drainage (AILLIOT, 1980) confirment largement la relation entre fertilisation et meilleure résistance à l'ennoyage. Une étude comparée du rapport coût/efficacité entre une fertilisation reñforcée, y compris par des moyens aériens pendant l'ennoyage, et l'installation d'un réseau de drainage pourrait être envisagée.

Recu le 15 juin 1984. Accepté le 11 avril 1985.

\section{REMERCIEMENTS}

Mme R. Zfiwer, M. G. Vuillemin et Mme M. Coupeal (Rhône-Poulenc Recherches, Centre de Recherches de la Croix de Berny) sont vivement remerciés pour leur assistance technique. 


\section{RÉFÉRENCES BIBLIOGRAPHIQUES}

Ailliot B., 1980. Drainage. C. R. de l'enquête 1979 ; fév. 1980. C. R. de l'enquête 1980, déc. 1980. SUAD Chartres, Chambre d'Agriculture d'Eure-et-Loir.

Dagneaud J. P., Tranchefort J., Couvreur F., 1981. Blé tendre d'hiver: une méthode opérationnelle de prévision des rendements. Perspectives agricoles, 48, 49-58.

Drew M. C., 1983. Plant injury and adaptation to oxygen deficiency in the root environment : review. Plant Soil, 75, 179-199.

Drew M. C., Sisworo E. J., Saker L. R., 1979. Alleviation of waterlogging damage to young barley plants by application of nitrate and a synthetic cytokinin, and comparison between the effects of waterlogging, nitrogen deficiency and root excision. New Phytol., 82, 315-329.

Duthion C., Mingeau M., 1976. Les réactions des plantes aux excès d'eau et leurs conséquences. Ann. agron., 27, 221-246.

Guérif M., 1983. Structure de la variabilité du rendement du blé d'hiver dans quelques départements céréaliers français. I. Variabilité entre années et entre départements du peuplement épi et du poids de grain par épi. Agronomie, 3, 917-923.

Guyot Ch., Prioul J. L., 1985. Correction par la fertilisation minérale des effets de l'ennoyage sur le blé d'hiver. II. Expérimentation en culture hydroponique. Agronomie, 5 (8), 751-759.

Jackson W. T., 1955. The role of adventitious roots in recovery of shoots following flooding of the original root systems. Amer. J. Bot., 42, 816-819.

Jackson M. B., Campbell D. J., 1979. Effects of benzyladenine and gibberellic acid on the responses of tomato plants to anaerobic root environments and to ethylene. New Phytol., 82, 331-340.

Masle-Meynard J., 1981. Elaboration du nombre d'épis d'un peuplement de blé d'hiver en situation de compétition pour l'azote. I.
Mise en évidence d'un stade critique pour la montée d'une talle. Agronomie, 1, 623-631.

Masle-Meynard J., 1982. Flaboration du nombre d'épis d'un peuplement de blé d'hiver en situation de compétition pour l'azote. II Modélisation du nombre d'épis. Agronomie, 2, 17-23.

Masle-Meynard J., Sébillote M., 1981. Etude de l'hétérogénéité d'un peuplement de blé d'hiver. II. Origine des différentes catégories d'individus du peuplement ; éléments de description de sa structure. Agronomie, 1, 217-223.

Morisset C., Raymond P., Mocquot B., Pradet A., 1982. Adaptation des végétaux à l'hypoxie et l'anoxie. Bull. Soc. Bot. Fr., 129, 73-89.

Shalhevet J., Zwerman P. J., 1962. Nitrogen response of corn under variable conditions of drainage. A lysimeter study. Soil Sci. 93, 172-182

Stengel P., Marty J. R., Bosc M., Blanchet R., 1972. Conséquences d'ennoyages temporaires liés à la microtopographie de sols peu perméables à l'égard de quelques cultures. C. R. Acad. Agric. Fr., 58, 92-100.

Trought M. C. T., Drew M. C., 1980a. The development of waterlogging damage in wheat seedlings (Triticum aestivum L.). I. Shoot and root growth in relation to changes in the concentrations of dissolved gases and solutes in the soil solution. Plant Soil, 54, 77-94.

Trought M. C. T., Drew M. C., 1980b. The development of waterlogging damage in wheat seedlings (Triticum aestivum L.). II. Accumulation and redistribution of nutrients by the shoot. Plant Soil, 56, 187-199.

Trought M. C. T., Drew M. C., 1982. Effects of waterlogging on young wheat plants (Triticum aestivum L.) and on soil solutes at different soil temperatures. Plant Soil, 69, 311-326. 